\title{
Correlation of Body Habitus with Severity of Obstructive Sleep Apnea: A Prospective Study from a Single Institution
}

\author{
${ }^{1}$ Renuka Balu, ${ }^{2}$ Uttumadathil G Unnikrishnan, ${ }^{3}$ Madhumita Kumar
}

\begin{abstract}
Objectives: To correlate body habitus with severity of obstructive sleep apnea (OSA) in Indian subjects.
\end{abstract}

Subjects: Prospective study. Consecutive patients with OSA during the period 2013 to 2016 were included.

Materials and methods: Body mass index (BMI), neck circumference (NC), and Epworth sleepiness scale (ESS) were recorded and their association with severity of OSA as graded by apnea-hypopnea index ( $\mathrm{AHI})$ was analyzed.

Results: Totally 100 patients were included; 91 were male. Mean age was $46.58 \pm 12.92$ years. Abnormal BMI, NC, and ESS were observed in $67.3,33.6$ and $52.7 \%$ respectively. Moderate and severe forms of OSA were found in 26 and $54 \%$ respectively. Severe OSA was more common in men (56 vs $33.3 \% ; p=0.15)$. There was a strong correlation of OSA with BMI $(r=0.378 ; p<0.001)$, NC $(r=0.502 ; p<0.001)$, and ESS $(r=0.304 ; p=0.002)$.

Conclusions: Obstructive sleep apnea in Indian patients has strong correlation with male sex and indices of body habitus.

Keywords: Apnea-hypopnea index, Body mass index, Neck circumference, Obstructive sleep apnea.

How to cite this article: Balu R, Unnikrishnan UG, Kumar M. Correlation of Body Habitus with Severity of Obstructive Sleep Apnea: A Prospective Study from a Single Institution. Indian Sleep Med 2017;12(2):7-11.

Source of support: Nil

Conflict of interest: None

\section{INTRODUCTION}

Obstructive sleep apnea hypopnea syndrome (OSAHS) is characterized by repetitive episodes of apnea and hypopnea during sleep. ${ }^{1}$ Initially described by Guilleminault et $\mathrm{al}^{2}$ in the 1970s, understanding of its pathogenesis has evolved over the past 40 years. The obstruction involves

\footnotetext{
${ }^{1}$ Clinical Assistant Professor, ${ }^{2}$ Lecturer, ${ }^{3}$ Professor and Head

${ }^{1,3}$ Department of ENT, Amrita Institute of Medical Sciences and Research Centre, Kochi, Kerala, India

${ }^{2}$ Department of Biostatistics, Amrita Institute of Medical Sciences and Research Centre, Kochi, Kerala, India

Corresponding Author: Renuka Balu, Clinical Assistant Professor, Amrita Institute of Medical Sciences and Research Centre, Kochi, Kerala, India, Phone: +919447909600 , e-mail: drrenukabalu@gmail.com
}

the upper airway (UAW), specifically the pharynx, because of imbalance between intrapharyngeal pressure and outward forces generated by pharyngeal soft tissue and muscles. In addition, specific anatomic abnormalities or a disproportional anatomy which can compromise pharyngeal lumen and size is present in patients with OSAHS. ${ }^{3}$ Changes in pharyngeal size and its configuration during sleep are a multifactorial consequence having direct participation of anatomical and neural disorders. ${ }^{4-6}$

The symptoms include excessive daytime sleepiness, nonrefreshing sleep, daytime fatigue, and decreased concentration, which may cause marked impairment in social or occupational functioning. ${ }^{7}$ In addition, the patients with OSA were shown to have an increased risk of cardiovascular disease, stroke, systemic hypertension, diabetes mellitus, depression, and impaired cognitive function. ${ }^{8,9}$ The gold standard for the diagnosis of OSA is overnight polysomnography (PSG). ${ }^{10}$ Documented risk factors for OSA include advanced age, male gender, systemic hypertension, large NC, and obesity. Previous studies demonstrated that large NC was a risk factor for severe OSA and the most important predictor of OSA. ${ }^{11-13}$ In addition, NC and BMI are highly correlated, and BMI has been the most widely used parameter to describe obesity. ${ }^{11}$ One study reported that a $10 \%$ weight gain predicted an approximate $32 \%$ increase in the AHI, and a $10 \%$ weight loss predicted a $26 \%$ decrease in $\mathrm{AHI}{ }^{14}$

The aim of the present study was to identify the correlation of severity of OSA as measured by AHI with indices of body habitus like BMI and NC in Indian patients.

\section{MATERIALS AND METHODS}

\section{Study Design and Setting}

This was a prospective study (2013-2016) conducted in a tertairy care teaching hospital in South India. A dedicated sleep apnea clinic was established in 2013 and the patients were evaluated according to a standard protocol and a database was maintained.

\section{Patients}

All patients referred to the sleep apnea clinic with chief complaint of snoring as witnessed by the sleep partner were included after evaluation with overnight PSG. 
Patients younger than 14 years and those with central apnea were excluded.

\section{Study Protocol}

All patients underwent a detailed clinical history taking and otolaryngological examination and completed a sleepiness scale (ESS). Clinical evaluation included evaluation for hypertension, cardiovascular disease, diabetes mellitus, hypothyroidism, nasal obstruction, and rhinitis. Physical examination assessed facial, oral cavity, and oropharynx characteristics and findings on flexible scopy for evaluation of level of collapse. A detailed assessment of the body habitus was performed including height $(\mathrm{cm})$, weight $(\mathrm{kg})$, and BMI calculated $\left(\mathrm{kg} / \mathrm{m}^{2}\right)$. Neck circumference $(\mathrm{cm})$ was measured at the level of cricothyroid membrane.

All patients underwent a detailed overnight PSG. The PSG parameters included were electroencephalogram, electrocardiogram, right and left eye movements, submental and anterior tibialis electromyogram, nasal and oral airflow limitations, thoracic and abdominal movements, and oxygen saturation by pulse oximetry. Apnea was defined as cessation of airflow for more than 10 seconds and hypopnea was defined as $\geq 50 \%$ decrease in airflow that persisted for more than 10 seconds accompanied by oxygen desaturation of $3 \%$ or greater or by arousal. ${ }^{15}$ The AHI was calculated as the total number of respiratory events (apnea plus hypopnea) per hour of sleep.

\section{Study Variables}

The BMI was categorized into three groups based on severity $-<25,25$ to 29.9, and $>30$. Epworth sleepiness scale was categorized into three groups: 0 to 8,9 to 15 , and $>15$. Neck circumference was grouped as either normal or abnormal. Neck circumference $>43 \mathrm{~cm}$ (males) and 38 $\mathrm{cm}$ (female) was considered abnormal.

\section{Outcome Variables}

Severity of OSA was graded by AHI as follows: $<5$ as normal, 5 to 15 mild, 15 to 30 moderate, and $>30$ as severe form of OSA. The correlation of severity of OSA with measures of body habitus (BMI and NC) as well as with the ESS was analyzed.

\section{Statistical Analysis}

The primary end point for this study was the presence of OSA as a dependent variable. The differences were analyzed using clinical variables, such as sex, age, BMI, NC, ESS as independent variables. Comparisons were performed using chi square test for categorical variables and Karl Pearson correlation coefficient for numerical variables. We also analyzed the difference in age, BMI, and NC according to the severity of OSA. All statistical tests were performed using IBM Statistical Package for the Social Sciences version 20. For all calculations a p-value of less than 0.05 was considered statistically significant. Categorical variables were presented as frequency and percentage. Numerical variables that had a normal distribution were presented as mean \pm standard deviation.

The study protocol was approved by the Institutional Ethics Committee.

\section{RESULTS}

During the period 2013-2016, a total of 199 patients were referred for evaluation to the sleep apnea clinic; 174 $(87.4 \%)$ were male. Of these, 100 patients (50.2\%) underwent PSG; 91 of these were male. The mean patient age was $46.58 \pm 12.92$ years. The mean BMI was $27.45 \mathrm{~kg} / \mathrm{m}^{2}$ $\left(19-45 \mathrm{~kg} / \mathrm{m}^{2}\right)$ and the mean $\mathrm{NC}$ was $41.75 \pm 3.05 \mathrm{~cm}$. Table 1 summarizes the baseline clinical characteritics of the study patients.

Abnormal BMI was observed in $67.3 \%$ of the study patients with $42.7 \%$ having BMI between 25 to 29.9 and $24.6 \%$ with $\mathrm{BMI}>30$. Epworth sleepiness scale score of 0 to 8 was observed in $47.3 \%$ patients; $44.4 \%$ had a score of 9 to 15 , while $8.3 \%$ had a score $>15$. The NC was abnormal in $33.6 \%$ of patients.

Based on analysis of AHI, 20, 26, and 54\% had mild, moderate, and severe OSA respectively. Table 2 summarizes the results of PSG-severity of AHI scales by sex. As summarized, severe forms of AHI were more commonly observed in men than in women (56 vs 33.3\%; $\mathrm{p}=0.15$ ).

Table 3 and Graphs 1 to 3 summarize the association of the study variables BMI, EPS, and NC with severity

Table 1: Baseline characteristics

\begin{tabular}{llll}
\hline Variables & Category & Frequency $(n=199)$ & Percentage \\
\hline Sex & Female & 25 & 12.6 \\
& Male & 174 & 87.4 \\
Smoking & Yes & 26 & 13.1 \\
& No & 137 & 68.8 \\
& Unknown & 36 & 18.1 \\
BMI & $<25$ & 35 & 17.6 \\
& $25-29.90$ & 85 & 42.7 \\
& $>30$ & 49 & 24.6 \\
& Unknown & 30 & 15.1 \\
\hline
\end{tabular}

Table 2: Severity of AHI assessed by PSG

\begin{tabular}{|c|c|c|c|c|c|c|c|}
\hline \multirow[b]{3}{*}{ Sex } & \multicolumn{6}{|c|}{$\mathrm{AHI}$} & \multirow[b]{3}{*}{$p$-value } \\
\hline & \multicolumn{2}{|c|}{$<15$} & \multicolumn{2}{|c|}{$16-30$} & \multicolumn{2}{|c|}{$>30$} & \\
\hline & $n$ & $\%$ & $n$ & $\%$ & $n$ & $\%$ & \\
\hline Female (9) & 4 & 44.5 & 2 & 22.2 & 3 & 33.3 & 0.150 \\
\hline Male (91) & 16 & 17.6 & 24 & 26.4 & 51 & 56 & \\
\hline
\end{tabular}




\begin{tabular}{|c|c|c|c|c|c|}
\hline \multirow[b]{2}{*}{ Variables } & \multirow[b]{2}{*}{ Category } & \multicolumn{3}{|c|}{$A H I$} & \multirow[b]{2}{*}{$p$-value } \\
\hline & & $\leq 15$ & $16-30$ & $>30$ & \\
\hline \multirow[t]{3}{*}{ BMI } & $<25$ & 33.3 & 26.7 & 40 & 0.027 \\
\hline & $25-29.9$ & 22.9 & 33.3 & 43.8 & \\
\hline & $\geq 30$ & 6.1 & 18.2 & 75.7 & \\
\hline \multirow[t]{3}{*}{ ESS } & $0-8$ & 35.9 & 25.6 & 38.5 & 0.022 \\
\hline & $9-15$ & 10.2 & 30.6 & 59.2 & \\
\hline & $>15$ & 11.1 & 11.1 & 77.8 & \\
\hline \multirow[t]{2}{*}{$\mathrm{NC}$} & Normal & 33.3 & 35.6 & 31.1 & 0.002 \\
\hline & Abnormal & 5.9 & 26.5 & 67.6 & \\
\hline
\end{tabular}

All figures are in percentages

of OSA as defined by AHI. There was a positive correlation of higher BMI with more severe AHI ( $p=0.027$; Graph 1). The AHI values of $>30$ were found in 43.8 and $75.7 \%$ of patients with BMI of 25 to 29.9 and $>30$ respectively (Table 3 ). Only $6.1 \%$ of patients in the BMI $>30$ category had $\mathrm{AHI}<15$. AHI $>30$ was significantly correlated with abnormal NC ( $\mathrm{p}=0.002$, Graph 2). In patients with abnormal $\mathrm{NC}, 67.6 \%$ of patients had $\mathrm{AHI}>30$ compared with $31.1 \%$ in patients with normal NC (Table 3). Epworth sleepiness scale score also positively correlated with severity of AHI ( $p=0.022$, Graph 3). The AHI $>30$ was found in $38.5,59.2$, and $77.8 \%$ of patients with ESS scores 0 to 8,9 to 15 , and $>15$ respectively (Table 3 ).

\section{DISCUSSION}

The major finding of this study was the strong correlation of indices of body habitus (BMI and NC) and the ESS with severity of OSA as assessed by AHI (Table 3, Graph 1 to 3). There was a linear correlation between indices of body habitus and OSA (Graphs 1 to 3). We also observed the association of male sex with more severe forms of OSA (Table 2). The strongest correlation for severe OSA was observed in patients with abnormal NC.

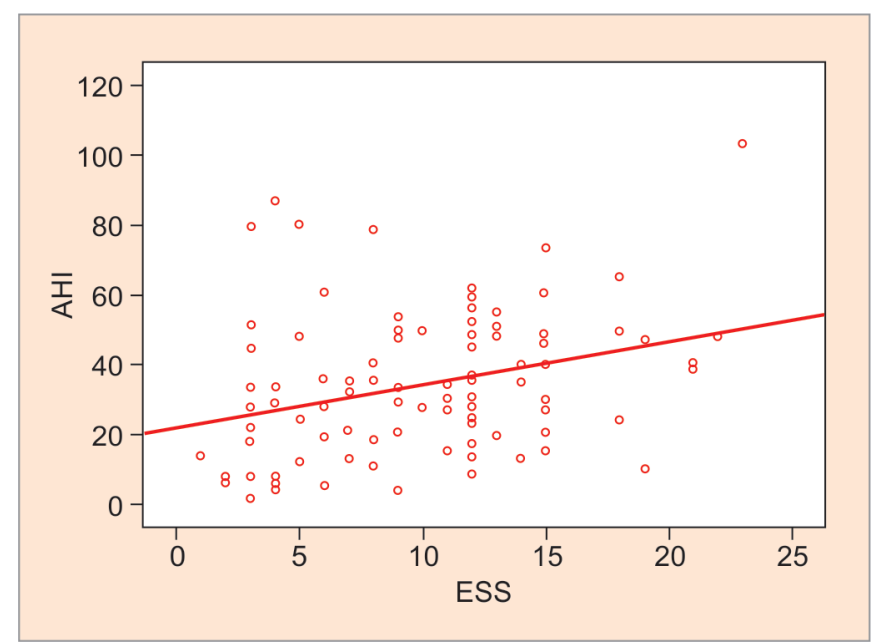

Graph 2: Correlation between $\mathrm{AHI}$ and ESS

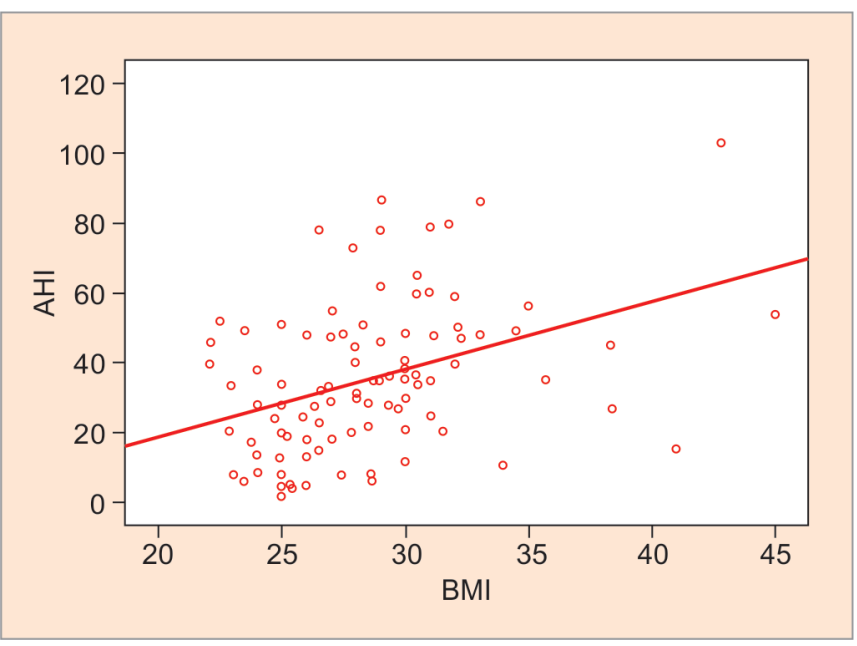

Graph 1: Correlation between $\mathrm{AHI}$ and BMI

Obesity is a major risk factor for OSA. ${ }^{16}$ Soft tissue enlargement around the UAW caused by obesity-related subcutaneous and periluminal fat deposits may alter the compliance of UAW walls and narrow the luminal area. Thus, this increased likelihood of airway collapse may explain the pathogenesis of OSA in obese patients. ${ }^{17,18}$ In addition, the pathogenesis of OSA may be mediated by secondary effects induced by obesity, such as inflammation, insulin resistance, visceral adiposity, and central neural mechanisms. ${ }^{19}$ Obesity also negatively affects lung function, which may exaggerate an existing airway disease and obese subjects also have increased respiratory resistance and reduced lung volumes.

In this study, we observed that an abnormal NC was more strongly associated with OSA than obesity (Table 3; Graph 3). This was reported in a previous study in Japanese subjects. ${ }^{13}$ A possible explanation for this finding is that localized adipose tissue distribution around the neck is more associated with OSA than general obesity and OSA is more vulnerable to the change in NC of Asian patients. ${ }^{20}$ In addition, NC is considered a marker of

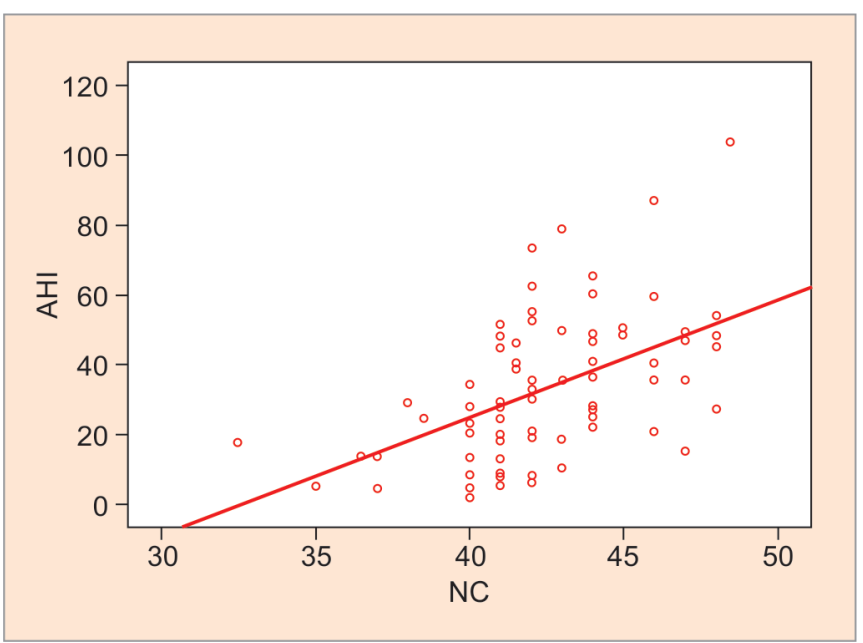

Graph 3: Correlation between AHI and NC 
central obesity and has been associated not only with OSA but also with increased cardiovascular risk and insulin levels. ${ }^{21}$ An increased NC has been suggested as a better sign of OSA than other clinical indices, ${ }^{22,23}$ and may be upto $77 \%$ sensitive and $82 \%$ specific for OSA in patients. Furthermore, combining NC with other features of sleep apnea might further increase its sensitivity and specificity, allowing the clinical diagnosis to be made. ${ }^{24}$

Although a relationship between the ESS score and AHI has been observed, ${ }^{25,26}$ a clear causative relationship has not been established. ${ }^{27}$ In our study, there was a strong trend toward higher AHI in the group with ESS $\geq$ 13 compared with ESS $\leq 6$ group and the difference is statistically significant. So repetitive intermittent nocturnal hypoxemia plays a major role in the pathogenesis of excessive daytime sleepiness in patients with OSA.

There are limitations to this study. Firstly, this was a cross-sectional study. We could not determine the actual effect of NC on the development and severity of OSA. Secondly, we enrolled only those patients who visited the sleep apnea clinic of a tertiary hospital and the possibility of referral bias is possible. Thus, it may not be possible to extrapolate our findings to the general population. Finally, we did not design interventions to improve the body habitus and study its impact on the severity of OSA on follow-up.

\section{CONCLUSION}

Obstructive sleep apnea in Indian patients has a strong correlation with male sex and indices of body habitus like $\mathrm{BMI}$ and NC and the ESS.

\section{REFERENCES}

1. Chung F, Yang Y, Liao P. Predictive performance of STOP-Bang score for obstructive sleep apnoea in obese patients. Obes Surg 2013 Dec;23(12);2050-2057.

2. Guilleminault C, Tilkian A, Dement WC. The sleep apnea syndromes. Annu Rev Med 1976;27:465-484.

3. Isono, S.; Remmers, JE. Anatomy and physiology of upper airway obstruction. In: Krygger MH, Roth T, Dement WC, editors. Principles and practice of sleep medicine, 2nd ed. Philadelphia, PA: WB Saunders; 1994. pp. 642-656.

4. Robinson, A.; Guilleminault, C. Obstructive sleep syndrome. In: Chokroverty S, editor. Sleep disorders medicine: basic science, technical considerations, and clinical aspects, 2nd ed. Woburn: Butterworth Heinemann; 1999. pp. 331-354.

5. SmirneS, IannacconeS, Ferini-Strambi L, Comola M, ColomboE, Nemni R. Muscle fibre type and habitual snoring. Lancet 1991;337:597-599.

6. Sher AE. Obstructive sleep apnoea syndrome: a complex disorder of the upper airway. Otolaryngol Clin North Am 1990 Aug;23(4):593-608.

7. Ogretmenoglu O, Suslu AE, Yucel OT, Onerci TM, Sahin A. Body fat composition: a predictive factor for obstructive sleep apnea. Laryngoscope 2005 Aug;115(8):1493-1498.
8. Vana KD, Silva GE, Goldberg R. Predictive abilities of the STOP-Bang and Epworth Sleepiness Scale in identifying sleep clinic patients at high risk for obstructive sleep apnea. Res Nurs Health 2013 Feb;36(1):84-94.

9. Farney RJ, Walker BS, Farney RM, Snow GL, Walker JM. The STOP-bang equivalent model and prediction of severity of obstructive sleep apnea; relation to polysomnographic measurements of the apnea/hypopnea index. J Clin Sleep Med 2011 Oct;7(5):459-465.

10. American Academy of Sleep Medicine. Sleep related breathing disorders in adults: recommendations for syndrome definitions and measurements techniques in clinical research. Sleep 1999 Aug;22(5):667-689.

11. Ahbab S, Ataoglu HE, Tuna M, Karasulu L, Çetin F, Temiz LU, Yenigün M. Neck circumference, metabolic syndrome and obstructive sleep apnoea syndrome; evaluation of possible linkage. Med Sci Monit 2013 Feb;19:111-117.

12. Flemons WW. Clinical practice. Obstructive sleep apnea. N Engl J Med 2002 Aug;347(7):498-504.

13. Kawaguchi $Y$, Fukumoto S, Inaba M, Koyama H, Shoji T, Shoji S, Nishizawa Y. Different impacts of neck circumference and visceral obesity on the severity of obstructive sleep apnea syndrome. Obesity (Silver Spring) 2011 Feb;19(2): 276-282.

14. Peppard PE, Young T, Palta M, Dempsey J, Skatrud J. Longitudinal study of moderate weight change and sleep disordered breathing. JAMA 2000 Dec;284(23):3015-3021.

15. Iber, C.; Ancoli-Israel, S.; Chesson, AL Jr.; Quan, SF. The AASM Manual for the scoring of sleep and associated events: rules terminology and technical specifications. Westchester, IL: American Academy of Sleep Medicine; 2007.

16. Plywaczewski R, Bielen P, Bednarek M, Jonczak L, Gorecka D, Sliwinski P. [Influence of neck circumference and body mass index on obstructive sleep apnoea severity in males]. Pneumonol Alergol Pol 2008;76(5):313-320.

17. Sakakibara $\mathrm{H}$, Tong $\mathrm{M}$, Matsushita K, Hirata M, Konishi $\mathrm{Y}$, Suetsugu S. Cephlometric abnormalities in non-obese and obese patients with obstructive sleep apnoea. Eur Respir J 1999 Feb;13(2):403-410.

18. Caples SM, Gami AS, Somers VK. Obstructive sleep apnoea. Ann Intern Med 2005 Feb;142(3):187-197.

19. Vgontzas AN. Does obesity play a major role in the pathogenesis of sleep apnoea and its associated manifestations via inflammation, visceral adiposity and insulin resistance? Arch Physiol Biochem 2008;114(4):211-223.

20. Soylu AC, Levent E, Sariman N, Yurtlu S, Alparslan S, Saygi A. Obstructive sleep apnea syndrome and anthropometric obesity indexes. Sleep Breath 2012 Dec;16(4):1151-1158.

21. Medeiros CA, Bruin VM, Castro-Silva C, Bruin PF. Neck circumference, a bedside clinical feature related to mortality of acute ischemic stroke. Rev Assoc Med Bras 2011 SeptOct;57(5):559-564.

22. Martins AB, Tufik S, Moura SM. Physiopathology of obstructive sleep apnoea-hypopnoea syndrome. J Bras Pneumol 2007;33(1):93-100.

23. Davies RJO, Stradling JR. The relationship between neck circumference, radiographic pharyngeal anatomy, and the obstructive sleep apnoea syndrome. Eur Respir J 1990 May;3(5):509-514.

24. Katz I, Stradling JR, Slutsky AS, Zamel N, Hoffstein V. Do patients with obstructive sleep apnoea have a thick neck? Am Rev Respir dis 1990 May;141(5 Pt 1):1228-1231. 
25. Sun Y, Ning Y, Huang L, Lei F, Li Z, Zhou G, Tang X. Polysomnographic characteristics of day time sleepiness in obstructive sleep apnoea syndrome. Sleep Breath 2012 Jun;16(2):375-381.

26. Chen R, Xiong KP, Lian YX, Huang JY, Zhao MY, Liu CF. Daytime sleepiness and its determining factors in Chinese obstructive sleep apnoea patients. Sleep Breath 2011;15(1): 129-135.

27. Mediano O, Barcelo A, de la Pena M, Gozal D, Agusti A, Barbe F. Daytime sleepiness and polysomnographic variables in sleep apnoea patients. Eur Respir J 2007 Jul;30(1):110-113. 